\title{
Localization of Massive and Massless Fermions on Two-Field Branes
}

\author{
Ali Farokhtabar' ${ }^{1}$ and Ali Tofighi ${ }^{2}$ \\ ${ }^{1}$ Young Researchers and Elite Club, Islamic Azad University, Babol Branch, Babol, Iran \\ ${ }^{2}$ Department of Physics, Faculty of Basic Science, University of Mazandaran, P.O. Box 47416-1467, Babolsar, Iran
}

Correspondence should be addressed to Ali Tofighi; a.tofighi@umz.ac.ir

Received 19 September 2016; Revised 21 December 2016; Accepted 4 January 2017; Published 26 March 2017

Academic Editor: Sally Seidel

Copyright (C) 2017 Ali Farokhtabar and Ali Tofighi. This is an open access article distributed under the Creative Commons Attribution License, which permits unrestricted use, distribution, and reproduction in any medium, provided the original work is properly cited. The publication of this article was funded by SCOAP ${ }^{3}$.

We study fermion localization and resonances on a special type of brane-world model supporting brane splitting. In such models one can construct multiwall branes which cause considerable simplification in the field equations. We use a polynomial superpotential to construct this brane. The suitable Yukawa coupling between the background scalar field and the localized fermion is determined. The massive fermion resonance spectrum is obtained. The number of resonances is increased for higher values of Yukawa coupling.

\section{Introduction}

Brane-world scenarios have attracted considerable attention in the literature during the last two decades because these models can address some important issues in theoretical physics problems such as hierarchy [1-3] and cosmological constant problem $[4,5]$. The branes in Randall and Sundrum (RS) models are fixed in some points along extra dimension and have a $\delta$ function form $[1,6]$. This brane-world model is very ideal and its formation has no dynamical mechanism. But for realistic models thickness of brane should be considered. By now several thick brane construction mechanisms have been developed such as thick branes generated from pure gravity [7-11], fermion self-interaction branes [12, 13], and thick brane scenarios with the gravity coupled to fivedimensional scalar fields [14-21]. In the last scenario, the scalar field configuration is usually a kink. It is found that a single kink becomes unstable when it moves in a discrete lattice with a large velocity while multikink solutions remain stable [22]. This phenomenon is associated with interaction between kink and radiation, and the resonances were observed experimentally [23, 24]. Furthermore, in cosmology we encounter models in which our universe is the result of continuous collision of branes and nucleation and therefore splitting of branes is a fundamental scenario in these models [25-28].
Therefore, the universal aspects of thick brane splitting in warped bulk are important. Such branes are constructed from a complex $\phi^{4}$ scalar field potential [29] or from a real $\phi^{6}$ scalar field potential [30]. These branes can be constructed from deformation of $\phi^{4}$ scalar field potential as well [31-33].

Recently, Dutra and coworkers proposed a new model of thick brane in which multibrane scenario arises from scalar field models generating usual kink solutions [34]. It suggests a special type of brane splitting. In this method superpotential function and warp factor will decompose in a special form and field equations will be simplified significantly. In this work we deal with this thick brane model which arises from polynomial superpotential.

The localization of spin $1 / 2$ fermions on the brane is very interesting and important. Usually, in five dimensions fermion does not have a normalizable zero mode without scalar-fermion coupling [35-43]. In five dimensions, with a Yukawa scalar-fermion coupling, there may exist a massless bound state and a continuous gapless spectrum of massive Kaluza-Klein (KK) states [39], while, in some of other brane models, there exist some discrete KK states and a continuous gapless mass spectrum [44].

This paper is organized as follows. In the next section, we present the brane model that is constructed in [34]. In Section 3 we investigate localization of the zero mode 
of the fermion field on the brane which is derived from a polynomial potential. In Section 4 we study localization of massive fermionic modes. Finally, in the last section we present our conclusions.

\section{The Model}

We consider the following action in which two scalar fields are coupled to gravity in 5 dimensions:

$$
\begin{aligned}
S= & \int d^{4} x d r \sqrt{-g}\left[\frac{1}{4} R-\frac{1}{2} \partial_{M} \phi_{1} \partial^{M} \phi_{1}-\frac{1}{2} \partial_{M} \phi_{2} \partial^{M} \phi_{2}\right. \\
& \left.-V\left(\phi_{1}, \phi_{2}\right)\right],
\end{aligned}
$$

where $g=\operatorname{det}\left(g_{M N}\right), M, N=0,1,2,3,5$. The coordinates on the brane are represented by $x^{\mu}(\mu=0,1,2,3)$ while the coordinate in the bulk is shown by $x^{5}=r$. The line element is written as

$$
d s^{2}=g_{M N} d x^{M} d x^{N}=e^{2 A(r)} \eta_{\mu \nu} d x^{\mu} d x^{\nu}+d r^{2}
$$

where $\eta_{\mu \nu}$ is the usual Minkowski metric with $\operatorname{diag}(-,+,+,+)$ and $e^{2 A(r)}$ is called the warp factor. For this brane-world scenario, the equations of motion are obtained as

$$
\begin{aligned}
\phi_{i}^{\prime \prime}+4 A^{\prime} \phi_{i}^{\prime} & =\frac{d V}{d \phi_{i}}, \quad(i=1,2), \\
A^{\prime \prime}+\frac{2}{3}\left(\phi_{1}^{\prime 2}+\phi_{2}^{\prime 2}\right) & =0, \\
A^{\prime 2}+\frac{1}{3} V\left(\phi_{1}, \phi_{2}\right) & =\frac{1}{6}\left(\phi_{1}^{\prime 2}+\phi_{2}^{\prime 2}\right),
\end{aligned}
$$

and the potential $V\left(\phi_{1}, \phi_{2}\right)$ can be written in terms of a superpotential $W\left(\phi_{1}, \phi_{2}\right)$ as

$$
V\left(\phi_{1}, \phi_{2}\right)=\frac{1}{2} \sum_{i=1}^{2}\left(\frac{\partial W}{\partial \phi_{i}}\right)^{2}-\frac{4}{3} W^{2} .
$$

Therefore, equations of motion can be reduced to the following first-order equations:

$$
\begin{aligned}
& \frac{d \phi_{i}}{d r}=\frac{\partial W}{\partial \phi_{i}}, \\
& \frac{d A}{d r}=-\frac{2}{3} W, \\
& (i=1,2) .
\end{aligned}
$$

But

$$
\begin{gathered}
W\left(\phi_{1}, \phi_{2}\right)=W_{1}\left(\phi_{1}\right)+W_{2}\left(\phi_{2}\right), \\
A(r)=A_{1}(r)+A_{2}(r) .
\end{gathered}
$$

The first-order equations in (5) are converted to

$$
\begin{aligned}
\frac{d \phi_{i}}{d r} & =\frac{\partial W_{i}\left(\phi_{i}\right)}{\partial \phi_{i}}, \\
\frac{d A_{i}}{d r}=-\frac{2}{3} W_{i}\left(\phi_{i}\right), & \\
& (i=1,2),
\end{aligned}
$$

for polynomial superpotential

$$
W_{i}\left(\phi_{i}\right)=\lambda_{i}\left(\phi_{i}-\frac{\phi_{i}^{3}}{3}\right), \quad(i=1,2)
$$

$\phi_{i}$ and $A_{i}$ are given by

$$
\begin{aligned}
\phi_{i}= & \tanh \left[\lambda_{i}\left(r-r_{i}\right)\right], \quad(i=1,2), \\
A(r)= & \frac{1}{9} \sum_{i=1}^{2}\left\{\tanh ^{2}\left[\lambda_{i}\left(\tilde{r}-r_{i}\right)\right]-\tanh ^{2}\left[\lambda_{i}\left(r-r_{i}\right)\right]\right\} \\
& -\frac{4}{9} \ln \left(\prod_{i=1}^{2} \frac{\operatorname{sech}\left[\lambda_{i}\left(\tilde{r}-r_{i}\right)\right]}{\operatorname{sech}\left[\lambda_{i}\left(r-r_{i}\right)\right]}\right),
\end{aligned}
$$

where $r_{i}$ is an integration constant, representing the center of the kink. For solitonic solutions we consider $\lambda_{i}>0$ and $\tilde{r}$ is defined as the average value of coordinates of center of the kinks

$$
\widetilde{r}=\frac{1}{2}\left(r_{1}+r_{2}\right)
$$

In order for this model to support brane splitting mechanism, we consider two symmetric kink solutions. Therefore, $\lambda_{1}=$ $\lambda_{2}=\lambda$ and $r_{1}=-r_{2}=a$. So $\tilde{r}=0$ and we can write (9) as

$$
\begin{aligned}
& \phi_{1}=\tanh [\lambda(r-a)], \\
& \phi_{2}=\tanh [\lambda(r+a)], \\
& A(r)=\frac{1}{9}\left\{2 \tanh ^{2}(\lambda a)-\tanh ^{2}[\lambda(r-a)]\right. \\
& \left.\quad-\tanh ^{2}[\lambda(r+a)]\right\}-\frac{4}{9} \\
& \quad \cdot \ln \left(\frac{\cosh [\lambda(r-a)] \cosh [\lambda(r+a)]}{\cosh ^{2}(\lambda a)}\right) .
\end{aligned}
$$

In Figure 1 we show the warp factor for different values of $a$. For $a=0$ we have a single brane that warp factor has a sharp peak. For $a>0$ a plateau is formed in the interior of the brane where the energy density vanishes. This is attributed to the presence of a new phase inside the brane. The plateau region in the brane grows when $a$ increases.

In the next section, we investigate localization of zero mode of the fermion on the brane. 


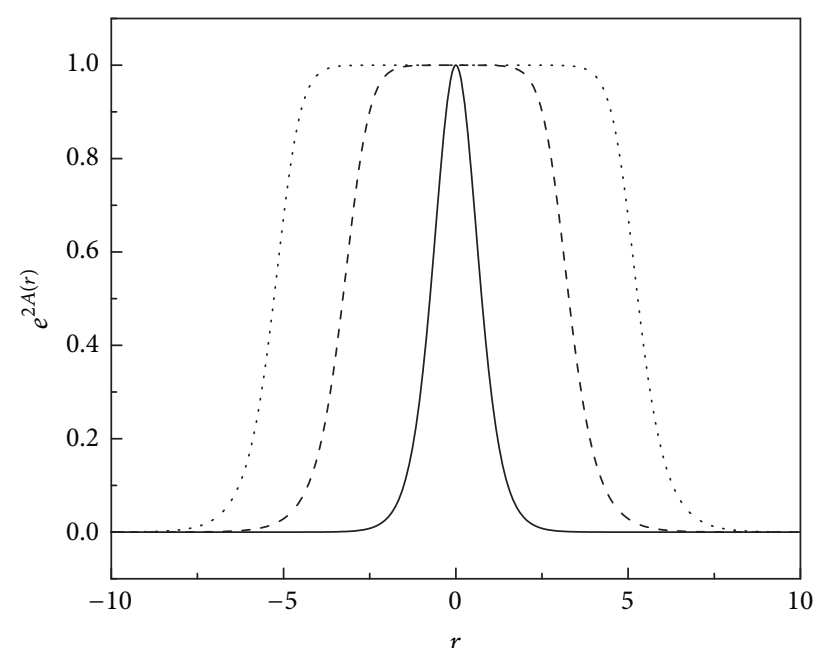

Figure 1: Plots of warp factor $e^{2 A(r)}$ for $a=0$ (solid line), $a=3.0$ (dashed line), and $a=5.0$ (dotted line) and we put $\lambda=1.0$.

\section{Localization of Zero Mode}

In order to get the mass-independent potential for $\mathrm{KK}$ modes of fermion, we perform the following coordinate transformation:

$$
d z=e^{-A} d r
$$

by this transformation we get a conformally flat metric

$$
d s^{2}=e^{2 A}\left(\eta_{\mu \nu} d x^{\mu} d x^{\nu}+d z^{2}\right) \text {. }
$$

In five dimensions, fermions are four component spinors and their Dirac structure can be described by $\Gamma^{M}=e^{M}{ }_{\bar{M}} \Gamma^{\bar{M}}$ with $e^{M} \bar{M}$ being the vielbein and $\left\{\Gamma^{M}, \Gamma^{N}\right\}=2 g^{M N} . \Gamma^{\bar{M}}$ are the gamma matrices in $5 \mathrm{D}$ flat space-time. The vielbein is given by

$$
e_{M}^{\bar{M}}=\left(\begin{array}{cc}
e^{A} \widehat{e}_{\mu}^{\bar{v}} & 0 \\
0 & e^{A}
\end{array}\right),
$$

$\Gamma^{M}=e^{-A}\left(\hat{e}^{\mu} \bar{\nu} \gamma^{\bar{\nu}}, \gamma^{5}\right)=e^{-A}\left(\gamma^{\mu}, \gamma^{5}\right)$, where $\gamma^{\mu}=\hat{e}^{\mu}{ }_{\bar{\nu}} \gamma^{\bar{\nu}}, \gamma^{\bar{\nu}}$ and $\gamma^{5}$ are the usual flat gamma matrices in the 4D Dirac representation. The action of a spin $1 / 2$ fermion coupled to the background scalar fields and gravity in five dimensions is

$$
S_{f}=\int d^{5} x \sqrt{-g}\left[\bar{\Psi} \Gamma^{M} D_{M} \Psi-\eta \bar{\Psi} F\left(\phi_{1}, \phi_{2}\right) \Psi\right],
$$

where the sign of coupling constant $\eta$ of the spinor $\Psi$ to scalars is arbitrary and represents a coupling to either kink or antikink domain wall. For definiteness, we shall consider only the case of a kink coupling and thus assume that $\eta>0$. $F\left(\phi_{1}, \phi_{2}\right)$ is a function of $\phi_{1}$ and $\phi_{2}$ determining the form of coupling between the fermion and scalars. The covariant derivative is defined as $D_{M}=\partial_{M}+\omega_{M}$ with the spin connection $\omega_{M}$ defined as $\omega_{M}=(1 / 4) \omega_{M}^{\bar{M}} \bar{N}_{\bar{M}} \Gamma_{\bar{N}}$ and $\omega_{M}^{\bar{M}} \bar{N}$ is

$$
\begin{aligned}
\omega_{M}^{\bar{M} \bar{N}}= & \frac{1}{2} e^{N \bar{M}}\left(\partial_{M} e_{N}^{\bar{N}}-\partial_{N} e_{M}^{\bar{N}}\right) \\
& -\frac{1}{2} e^{N \bar{N}}\left(\partial_{M} e_{N}^{\bar{M}}-\partial_{N} e_{M}^{\bar{M}}\right) \\
& -\frac{1}{2} e^{P \bar{M}} e^{Q \bar{N}}\left(\partial_{P} e_{\mathrm{Q} \bar{R}}-\partial_{\mathrm{Q}} e_{P \bar{R}}\right) e_{M}{ }^{\bar{R}} .
\end{aligned}
$$

The nonvanishing components of $\omega_{M}$ are

$$
\omega_{\mu}=\frac{1}{2} A^{\prime} \gamma_{\mu} \gamma_{5}+\widehat{\omega}_{\mu}
$$

where the prime denotes the derivative with respect to $z$ and $\widehat{\omega}_{\mu}=(1 / 4) \bar{\omega}_{\mu}^{\bar{\mu}} \Gamma_{\bar{\mu}} \Gamma_{\bar{\nu}}$ is the spin connection derived from the metric $\widehat{g}_{\mu \nu}(x)=\widehat{e}_{\mu}{ }^{\bar{\mu}}(x) \widehat{e}_{\nu}{ }^{\bar{\nu}}(x) \eta_{\bar{\mu} \bar{\nu}}$. Here we neglect the backreaction from the fermion on the brane solution and the scalar field is considered to be unchanged. Thus, by using the metric (13), the equation of motion for fermion is

$$
\begin{aligned}
& {\left[\gamma^{\mu}\left(\partial_{\mu}+\widehat{\omega}_{\mu}\right)+\gamma^{5}\left(\partial_{z}+2 \partial_{z} A\right)-\eta e^{A} F\left(\phi_{1}, \phi_{2}\right)\right] \Psi} \\
& \quad=0
\end{aligned}
$$

where $\gamma^{\mu}\left(\partial_{\mu}+\widehat{\omega}_{\mu}\right)$ is the Dirac operator on the brane.

For solving this equation, we separate variables with KK and chiral decomposition $[44,45]$

$$
\Psi(x, z)=e^{-2 A} \sum_{n}\left(\psi_{L n}(x) f_{L n}(z)+\psi_{R n}(x) f_{R n}(z)\right) .
$$

The $4 \mathrm{D}$ left-handed and right-handed fermions satisfy the Dirac equations

$$
\begin{aligned}
& \gamma^{\mu}\left(\partial_{\mu}+\widehat{\omega}_{\mu}\right) \psi_{L n}=m_{n} \psi_{R n}, \\
& \gamma^{\mu}\left(\partial_{\mu}+\widehat{\omega}_{\mu}\right) \psi_{R n}=m_{n} \psi_{L n},
\end{aligned}
$$

while the KK modes satisfy

$$
\begin{aligned}
& \left\{\partial_{z}+2 \partial_{z} A+\eta e^{A} F\left(\phi_{1}, \phi_{2}\right)\right\} f_{L n}(z)=m_{n} f_{R n}(z), \\
& \left\{\partial_{z}+2 \partial_{z} A-\eta e^{A} F\left(\phi_{1}, \phi_{2}\right)\right\} f_{R n}(z)=-m_{n} f_{L n}(z),
\end{aligned}
$$

with the following orthonormality condition

$$
\begin{aligned}
& \int_{-\infty}^{\infty} d z f_{L n} f_{L m}=\int_{-\infty}^{\infty} d z f_{R n} f_{R m}=\delta_{n m} \\
& \int_{-\infty}^{\infty} d z f_{L n} f_{R m}=0
\end{aligned}
$$

and the Schrödinger-like equations are obtained:

$$
\begin{aligned}
& {\left[-\partial_{z}^{2}+V_{L}(z)\right] f_{L n}=m_{n}^{2} f_{L n},} \\
& {\left[-\partial_{z}^{2}+V_{R}(z)\right] f_{R n}=m_{n}^{2} f_{R n},}
\end{aligned}
$$


where the effective potentials are given by

$$
\begin{aligned}
& V_{L}(z)=\eta^{2} e^{2 A} F^{2}\left(\phi_{1}, \phi_{2}\right)-\eta \partial_{z}\left[e^{A} F\left(\phi_{1}, \phi_{2}\right)\right], \\
& V_{R}(z)=\eta^{2} e^{2 A} F^{2}\left(\phi_{1}, \phi_{2}\right)+\eta \partial_{z}\left[e^{A} F\left(\phi_{1}, \phi_{2}\right)\right] .
\end{aligned}
$$

Because of the complexity of $A(r)$, we can not use relation (12) to obtain analytical form of $z(r)$. Therefore we apply numerical method to get pair of $(r, z)$ :

$$
\begin{gathered}
\partial_{z} A=e^{A(r)} \partial_{r} A, \\
\partial_{z} F=e^{A(r)} \partial_{r} F .
\end{gathered}
$$

By these relations, we can rewrite the potentials as a function of $r$ :

$$
\begin{aligned}
& V_{L}(z(r))=\eta e^{2 A}\left[\eta F^{2}-\partial_{r} F-F \partial_{r} A(r)\right], \\
& V_{R}(z(r))=\eta e^{2 A}\left[\eta F^{2}+\partial_{r} F+F \partial_{r} A(r)\right] .
\end{aligned}
$$

By substituting $m_{n}=0$ in (21), the left-handed and righthanded zero modes of fermions are obtained as

$$
\begin{aligned}
& f_{L 0} \propto \exp \left[-\eta \int_{0}^{z} d z^{\prime} e^{A\left(z^{\prime}\right)} F\left(\phi_{1}, \phi_{2}\right)\right], \\
& f_{R 0} \propto \exp \left[\eta \int_{0}^{z} d z^{\prime} e^{A\left(z^{\prime}\right)} F\left(\phi_{1}, \phi_{2}\right)\right] .
\end{aligned}
$$

The normalization condition for localization of zero mode of left-handed fermions on the brane is

$$
\begin{aligned}
& \int_{-\infty}^{\infty} d z \exp \left(-2 \eta \int_{0}^{z} d z^{\prime} e^{-A\left(z^{\prime}\right)} F\left(\phi_{1}\left(z^{\prime}\right), \phi_{2}\left(z^{\prime}\right)\right)\right) \\
& \quad<\infty,
\end{aligned}
$$

and in $r$ coordinate, we have

$$
\int_{-\infty}^{\infty} d r \exp \left(-A(r)-2 \eta \int_{0}^{r} d r^{\prime} F\left(\phi_{1}\left(r^{\prime}\right), \phi_{2}\left(r^{\prime}\right)\right)\right)
$$

$$
<\infty \text {. }
$$

For right-handed fermion this condition is achieved by replacing $\eta \rightarrow-\eta$. For studying localization of zero mode of fermion on the brane, we should determine the suitable form of $F\left(\phi_{1}, \phi_{2}\right)$. The procedure for determining $F\left(\phi_{1}, \phi_{2}\right)$ in this paper is similar to the methods used in $[46,47]$. In the following subsections we try to determine this function by using the normalization condition.

3.1. $F=\phi_{1} \phi_{2}$. The integrand in (30) can be expressed as

$$
\begin{aligned}
I_{0} & \equiv \exp \left[-\frac{1}{9}\left\{2 \tanh ^{2}(\lambda a)-\tanh ^{2}[\lambda(r-a)]\right.\right. \\
& \left.\left.-\tanh ^{2}[\lambda(r+a)]\right\}-2 \eta r\right] \\
& \times\left(\frac{\cosh [\lambda(r-a)] \cosh [\lambda(r+a)]}{\cosh ^{2}(\lambda a)}\right)^{4 / 9} \\
& \cdot\left(\frac{1+e^{-2 \lambda(r-a)}}{1+e^{-2 \lambda(r+a)}}\right)^{-2 \eta / \lambda} .
\end{aligned}
$$

Since $\eta>0$, when $r \rightarrow-\infty$ we have

$$
I_{0} \longrightarrow \exp \left(-\frac{8}{9}-2 \eta r\right) \longrightarrow \infty
$$

therefore, the integral in (30) is divergent. So the left-handed fermion zero mode can not be localized on the brane. On the other hand, with $\eta \rightarrow-\eta$, we can see that the zero mode of right-handed fermions can not be localized on the brane too.

3.2. $F=\phi_{1}-\phi_{2}$. The integrand in (30) is written as

$$
\begin{aligned}
I_{1} & \equiv \exp \left[-\frac{1}{9}\left\{2 \tanh ^{2}(\lambda a)-\tanh ^{2}[\lambda(r-a)]\right.\right. \\
& \left.\left.-\tanh ^{2}[\lambda(r+a)]\right\}\right] \\
& \times\left(\frac{\cosh [\lambda(r-a)] \cosh [\lambda(r+a)]}{\cosh ^{2}(\lambda a)}\right)^{4 / 9} \\
& \cdot\left(\frac{\cosh [\lambda(r-a)]}{\cosh [\lambda(r+a)]}\right)^{-2 \eta / \lambda}
\end{aligned}
$$

from which we have

$$
I_{1} \longrightarrow \exp \left( \pm \frac{8}{9} r\right) \longrightarrow \infty, \quad \text { when } r \longrightarrow \pm \infty .
$$

Hence, left-handed zero mode can not be localized on the brane in this case. Moreover, because $I_{1}$ is independent of $\eta$, therefore, we can conclude that right-handed fermion can not be localized on the brane.

3.3. $F=\phi_{1}+\phi_{2}$. The integrand in (30) is expressed as

$$
\begin{aligned}
I_{2} & \equiv \exp \left[-\frac{1}{9}\left\{2 \tanh ^{2}(\lambda a)-\tanh ^{2}[\lambda(r-a)]\right.\right. \\
& \left.\left.-\tanh ^{2}[\lambda(r+a)]\right\}\right] \\
& \times\left(\frac{\cosh [\lambda(r-a)] \cosh [\lambda(r+a)]}{\cosh ^{2}(\lambda a)}\right)^{4 / 9-2 \eta / \lambda} ;
\end{aligned}
$$

therefore, we have

$$
I_{2} \longrightarrow \exp \left[ \pm\left(\frac{8}{9} r-\frac{4 \eta r}{\lambda}\right)\right], \quad \text { when } r \longrightarrow \pm \infty
$$

So the normalization condition for localization of left-handed fermion zero mode is

$$
\eta>\frac{2 \lambda}{9}
$$

By changing $\eta \rightarrow-\eta$ one can find the zero mode of the righthanded fermion; however this mode can not be localized on the brane. 
The effective potentials for left-handed and right-handed KK fermion have the form

$$
\begin{aligned}
V_{L} & =\eta \exp \left[\frac { 2 } { 9 } \left\{2 \tanh ^{2}(\lambda a)-\tanh ^{2}[\lambda(r-a)]\right.\right. \\
& \left.\left.-\tanh ^{2}[\lambda(r+a)]\right\}\right] \\
& \times\left(\frac{\cosh [\lambda(r-a)] \cosh [\lambda(r+a)]}{\cosh ^{2}(\lambda a)}\right)^{-8 / 9} \\
& \cdot\left\{\eta(\tanh [\lambda(r-a)]+\tanh [\lambda(r+a)])^{2}\right. \\
& -\lambda\left(\operatorname{sech}^{2}[\lambda(r-a)]+\operatorname{sech}^{2}[\lambda(r+a)]\right. \\
& +\frac{2}{3}\left(\tanh ^{2}[\lambda(r-a)]+\tanh ^{2}[\lambda(r+a)]\right) \\
& \times\left\{\tanh ^{2}[\lambda(r-a)]+\tanh ^{2}[\lambda(r+a)]\right. \\
& \left.\left.-\frac{\tanh ^{3}[\lambda(r-a)]+\tanh ^{3}[\lambda(r+a)]}{3}\right\}\right), \\
V_{R} & =\left.V_{L}\right|_{\eta \rightarrow-\eta}
\end{aligned}
$$

The values of potential in $r$ or $z=0$ and $r$ or $z \rightarrow \pm \infty$ are

$$
\begin{aligned}
V_{L}(0) & =-V_{R}(0)=-2 \eta \lambda \operatorname{sech}^{2}(\lambda a), \\
V_{L}( \pm \infty) & =V_{R}( \pm \infty)=0 .
\end{aligned}
$$

It can be seen that the asymptotic behaviors of two potentials are the same when $r \rightarrow \pm \infty$, but opposite at the origin, $z=0$. This reveals that only one of the massless left or right chiral fermions can be localized on the brane. The shapes of effective potentials are shown in Figure 2. The form of $V_{L}(z)$ is volcano type and therefore, there is no mass gap between the zero mode and KK excitation modes. On the other hand, $V_{R}(z)$ is always positive at the brane location. We know that this type of potential can not trap any bound state of right-handed fermion and there is no zero mode of right-handed fermion. This is consistent with our pervious knowledge that only one chirality of massless fermion can exist.

The zero mode of left-handed fermion is written as

$$
\begin{aligned}
f_{L 0}(z) & \propto \exp \left(-\eta \int_{0}^{z} d z^{\prime} e^{A\left(z^{\prime}\right)}\left[\phi_{1}\left(z^{\prime}\right)+\phi_{2}\left(z^{\prime}\right)\right]\right) \\
& =\exp \left(-\eta \int_{0}^{r} d r^{\prime}\left[\phi_{1}\left(r^{\prime}\right)+\phi_{2}\left(r^{\prime}\right)\right]\right) \\
& =(\cosh [\lambda(r-a)] \cosh [\lambda(r+a)])^{-2 \eta / \lambda} .
\end{aligned}
$$

Figure 3 shows the form of fermion zero modes on the brane. One can see that the width of the function is increased with splitting.
3.4. The Case $F=\phi_{1}+\beta \phi_{2}$. The integrand in (30) is expressed as

$$
\begin{aligned}
I_{3} & \equiv \exp \left[-\frac{1}{9}\left\{2 \tanh ^{2}(\lambda a)-\tanh ^{2}[\lambda(r-a)]\right.\right. \\
& \left.\left.-\tanh ^{2}[\lambda(r+a)]\right\}\right] \times\left(\frac{\cosh [\lambda(r-a)]}{\cosh (\lambda a)}\right)^{4 / 9-2 \eta / \lambda} \\
& \cdot\left(\frac{\cosh [\lambda(r+a)]}{\cosh (\lambda a)}\right)^{4 / 9-\beta(2 \eta / \lambda)} .
\end{aligned}
$$

Therefore, we have

$$
I_{3} \longrightarrow \exp \left[ \pm\left(\frac{8}{9} r-\frac{2(\beta+1) \eta}{\lambda} r\right)\right]
$$

when $r \longrightarrow \pm \infty$.

So the normalization condition becomes

$$
\eta>\frac{4 \lambda}{9(\beta+1)}, \quad \beta \neq-1 .
$$

For $\beta=-1$ the relation (42) is reduced to (34). Hence, lefthanded zero mode can not be localized on the brane. For $\beta=$ 0 we have $\eta>4 \lambda / 9$. This means that coupling of fermion to every subbrane can localize zero mode on the brane. For the case $\beta=1$ the normalization condition is reduced to (37). The zero mode of left-handed fermion is turned out to be

$$
\begin{aligned}
f_{L 0}(z) & \propto \exp \left(-\eta \int_{0}^{r} d r^{\prime}\left[\phi_{1}\left(r^{\prime}\right)+\beta \phi_{2}\left(r^{\prime}\right)\right]\right) \\
& =\cosh [\lambda(r-a)]^{-2 \eta / \lambda} \cosh [\lambda(r+a)]^{-2 \beta \eta / \lambda} .
\end{aligned}
$$

The explicit forms of the potentials are

$$
\begin{aligned}
V_{L} & =\eta \exp \left[\frac { 2 } { 9 } \left\{2 \tanh ^{2}(\lambda a)-\tanh ^{2}[\lambda(r-a)]\right.\right. \\
& \left.\left.-\tanh ^{2}[\lambda(r+a)]\right\}\right] \\
& \times\left(\frac{\cosh [\lambda(r-a)] \cosh [\lambda(r+a)]}{\cosh ^{2}(\lambda a)}\right)^{-8 / 9} \\
& \cdot\left\{\eta(\tanh [\lambda(r-a)]+\beta \tanh [\lambda(r+a)])^{2}\right. \\
& -\lambda\left(\operatorname{sech}^{2}[\lambda(r-a)]+\beta^{2} \operatorname{sech}^{2}[\lambda(r+a)]\right. \\
& +\frac{2}{3}\left(\tanh ^{2}[\lambda(r-a)]+\beta \tanh ^{2}[\lambda(r+a)]\right) \\
& \times\left\{\tanh ^{2}[\lambda(r-a)]+\tanh ^{2}[\lambda(r+a)]\right. \\
& \left.\left.-\frac{\tanh [\lambda(r-a)]+\tanh ^{3}[\lambda(r+a)]}{3}\right\}\right), \\
V_{R} & =\left.V_{L}\right|_{\eta \rightarrow-\eta} .
\end{aligned}
$$




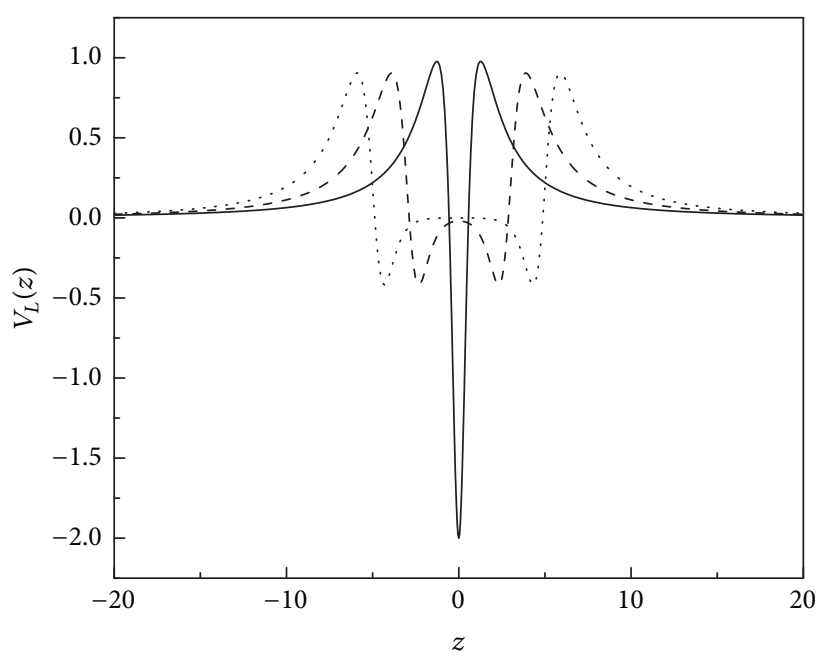

(a)

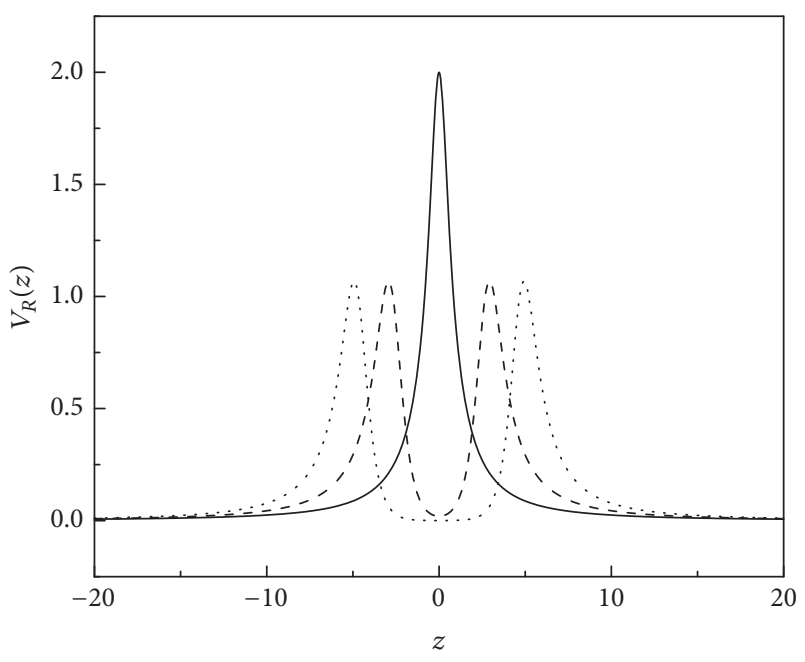

(b)

Figure 2: The shapes of potentials: (a) $V_{L}(z)$ and (b) $V_{R}(z), a=0$ (solid line), $a=3$ (dashed line), and $a=5$ (dotted line).

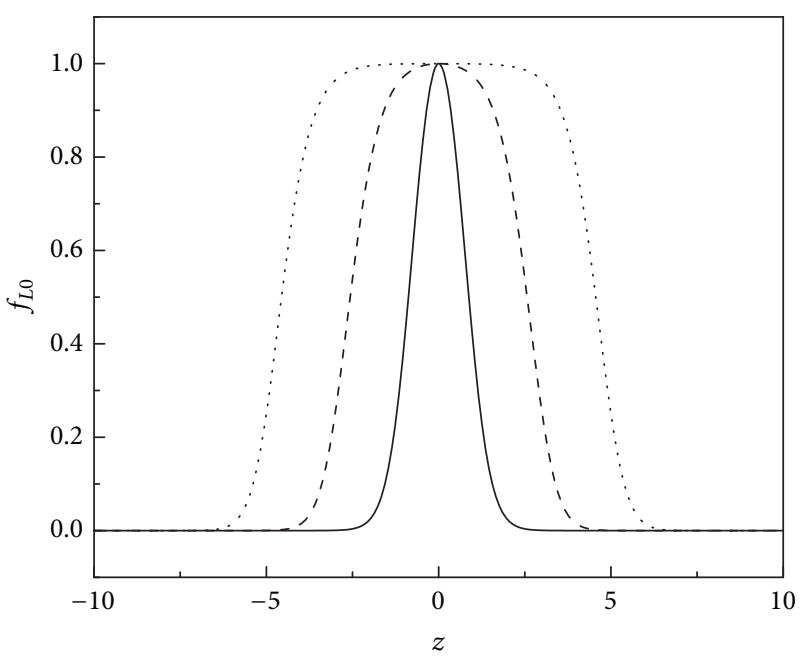

FIGURE 3: Fermion zero modes localized on the brane: $a=1$ (solid line), $a=3$ (dashed line), and $a=5$ (dotted line).

The asymptotic behaviors of the potentials are

$$
\begin{aligned}
& V_{L}(0)=\eta\left\{\eta(\beta-1)^{2}\right. \\
& \left.-\left[\eta(\beta-1)^{2}+\lambda\left(\beta^{2}+1\right)\right] \operatorname{sech}^{2}(\lambda a)\right\}, \\
& V_{R}(0)=\eta\left\{\eta(\beta-1)^{2}\right. \\
& \left.\quad-\left[\eta(\beta-1)^{2}-\lambda\left(\beta^{2}+1\right)\right] \operatorname{sech}^{2}(\lambda a)\right\}, \\
& V_{L}( \pm \infty)=V_{R}( \pm \infty)=0 .
\end{aligned}
$$

It is realized that $V_{L}(0)$ can be negative or positive, while, for $\eta>0$ and $\lambda>0, V_{R}(0)$ is always greater than $V_{L}(0)$. This means that localization of zero mode of the left-handed fermion is more supported.

\section{Resonances of Massive Modes}

We can rewrite (23) and (24) as

$$
\begin{aligned}
& Q^{\dagger} Q f_{L n}=\left(-\partial_{z}+\eta F e^{A}\right)\left(\partial_{z}+\eta F e^{A}\right) f_{L n}=m^{2} f_{L n} \\
& Q Q^{\dagger} f_{R n}=\left(\partial_{z}+\eta F e^{A}\right)\left(-\partial_{z}+\eta F e^{A}\right) f_{R n}=m^{2} f_{R n} .
\end{aligned}
$$

From these equations, we can see that the tachyonic modes in spectrum are excluded. With converting the equations of motion for fermion to Schrödinger-like equations, we can present a quantum mechanical interpretation for $f_{L n}$ and $f_{R n}$. By studying resonant modes we are able to obtain information about the coupling between massive modes and the brane.

In order to derive KK modes from (23) and (24) we apply relative probability method [48-50]. Since (23) and (24) are Schrödinger-like, we can interpret normalized $\left|f_{L, R}(z)\right|^{2}$ as the probability of finding massive KK modes on the brane. But the massive modes can not be normalized because they are oscillating when far away from brane along extra dimension. Therefore, the relative probability function is defined as [48]

$$
P_{L n, R n}(m)=\frac{\int_{-z_{b}}^{z_{b}} d z\left|f_{L n, R n}(z)\right|^{2}}{\int_{-z_{\max }}^{z_{\max }} d z\left|f_{L n, R n}(z)\right|^{2}},
$$

where $2 z_{b}$ is brane thickness approximately and $z_{\max }=10 z_{b}$ here. $f_{L n, R n}(z)$ is solution of (23) or (24) with two boundary conditions:

$$
\begin{aligned}
& f_{L n, R n}(0)=1, \\
& f_{L n, R n}^{\prime}(0)=0,
\end{aligned}
$$

for even parity, and

$$
\begin{aligned}
& f_{L n, R n}(0)=0, \\
& f_{L n, R n}^{\prime}(0)=1,
\end{aligned}
$$



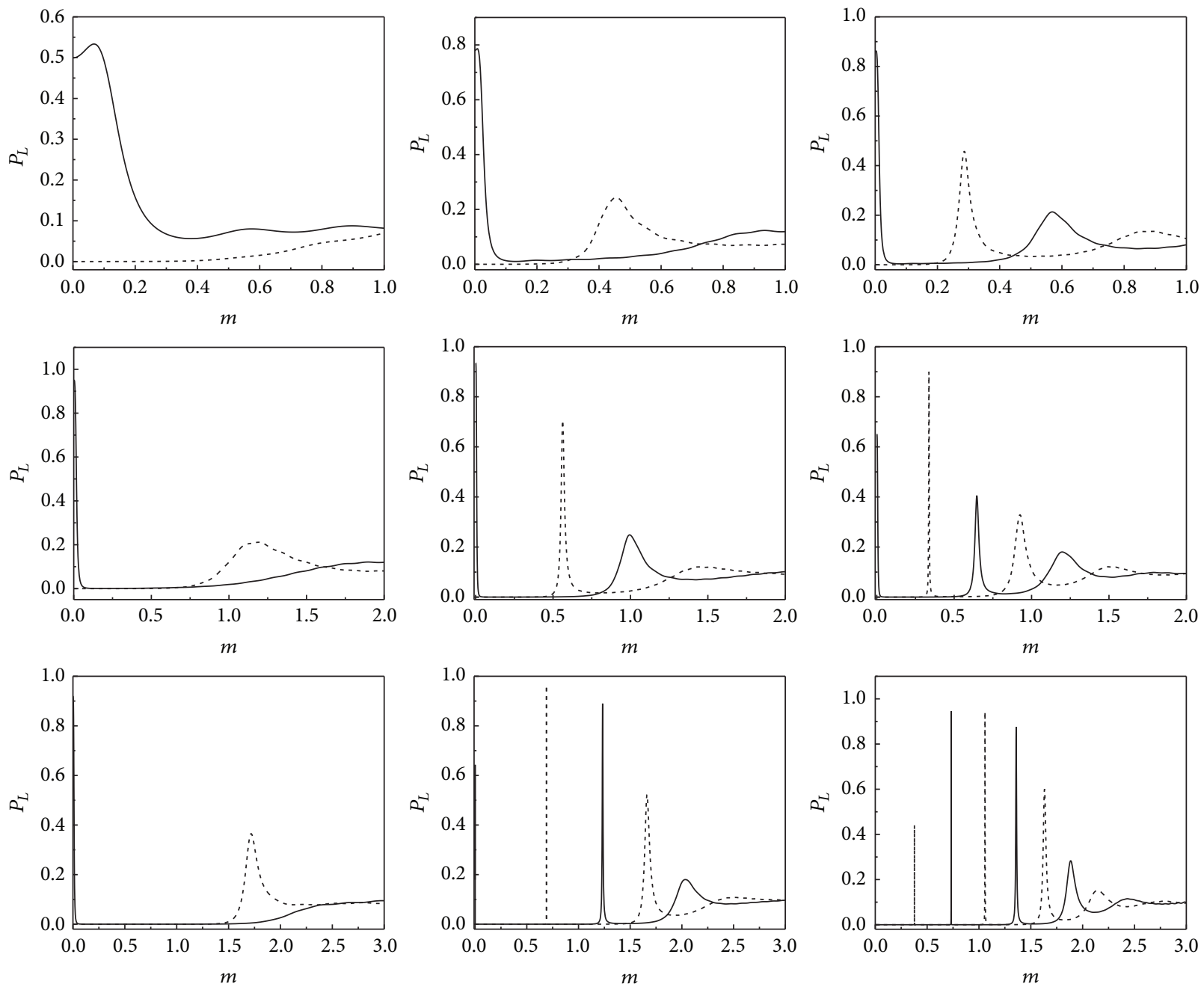

FIGURE 4: Plots of relative probability for left-handed fermion resonances with $a=1.0$ (left column), $a=3.0$ (middle column), and $a=5.0$ (right column). Coupling constants are $\eta=0.5$ (first raw), $\eta=1.0$ (second raw), and $\eta=2.0$ (third raw). Solid lines and dashed lines correspond to even and odd parity, respectively. In all cases $\lambda=1.0$.

for odd parity. If $m^{2} \gg V_{L, R \max }, f_{L, R}$ will be approximately a plane wave with $P_{L, R}(m)=z_{b} / z_{\max }=0.1$. For simplicity we have chosen $\beta=1$. Figures 4 and 5 show plots of the relative probability for different values of $a$ and $\eta$ for lefthanded and right-handed fermions, respectively. The masses of resonances are presented in Table 1 . The fermion resonant wave functions for $\eta=1, \lambda=1$, and $a=5.0$ are shown in Figure 6.

From the figures, we can see that the spectra of massive KK modes of left-handed and right-handed fermions are almost the same which reveals that a Dirac fermion is composed from left-handed and right-handed bound KK modes. Furthermore for fixed value of $a$ the larger the value of coupling parameter, the larger the number of resonances. Also for fixed value of $\eta$, the larger value of $a$ leads to larger number of peaks. The first peak is the most narrow and the resonances will become broader with increasing $m$. This means that first resonance has larger lifetime and the lifetime declines with increasing the mass of peak.
We can also see from figures that there are successively even and odd parity wave functions for left and right chiral modes with the same values of $\mathrm{m}^{2}$. In other words, the zero mode is beginning of left chirality with even parity spectra; therefore the two first resonances (if exist) with the same $\mathrm{m}^{2}$ are odd parity left chiral mode and even parity right chiral mode. Next the two second resonances (if exist) with the same $\mathrm{m}^{2}$ are even parity left chiral mode and odd parity right chiral mode.

From Figures 4 and 5 we can estimate the lifetime of every resonance as the reciprocal of width of peak at half of the height. With this definition we can find visually that heavier resonances are broader and they have shorter lifetimes. For evaluating this fact, we determine approximately the lifetimes of resonances of right-handed fermions for $\lambda=1.0, \eta=1.0$, and $a=5.0$ for instance. From Figure 5 we can see that in this case we have 4 resonances. The lifetimes of resonances are $\tau_{1}=242.1894$ for $m_{1}=0.327121, \tau_{2}=41.3873$ for $m_{2}=0.645689, \tau_{3}=12.4500$ for $m_{3}=0.924378$, and 

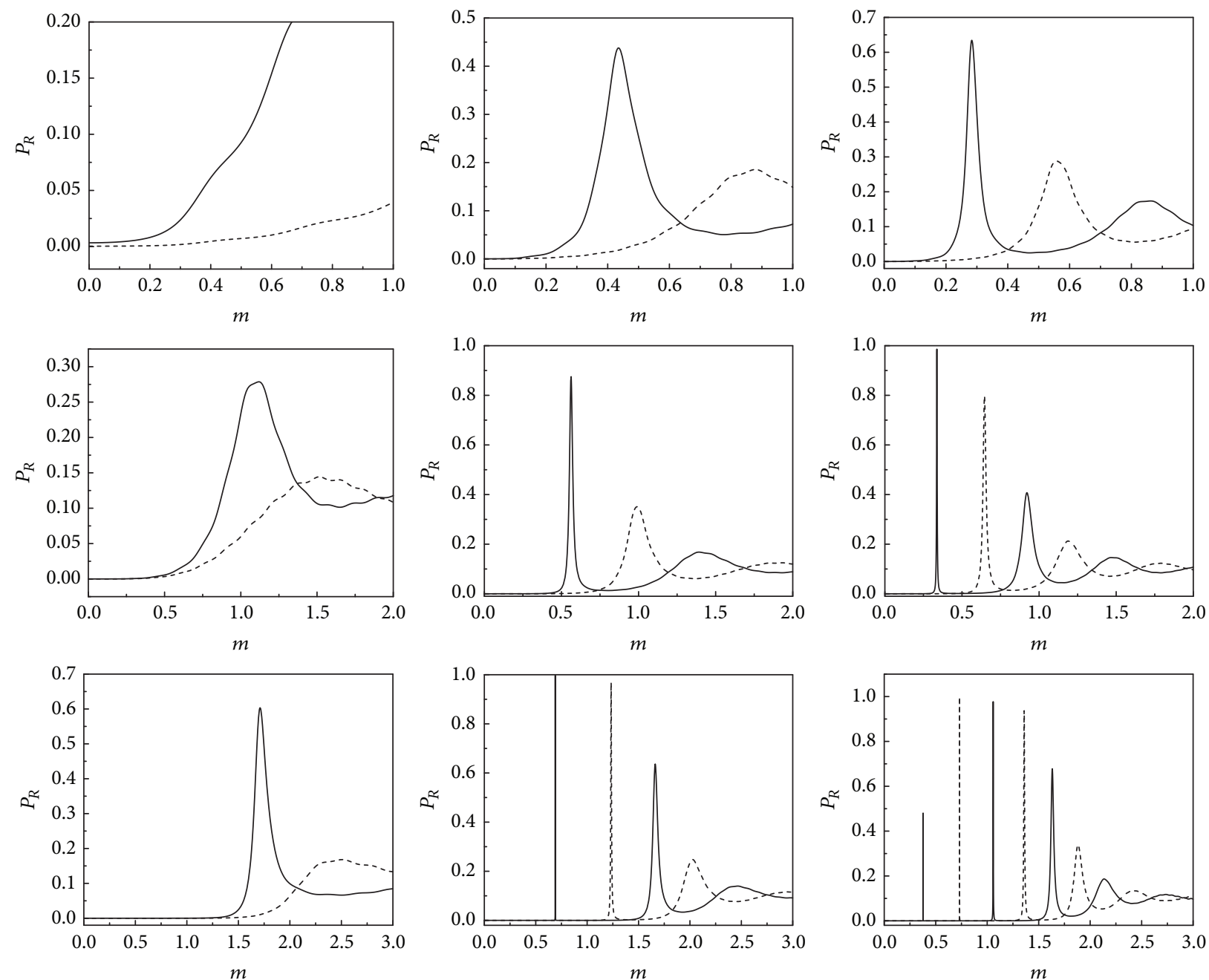

FIGURE 5: Plots of relative probability for right-handed fermion resonances with $a=1.0$ (left column), $a=3.0$ (middle column), and $a=5.0$ (right column). Coupling constants are $\eta=0.5$ (first raw), $\eta=1.0$ (second raw), and $\eta=2.0$ (third raw). Solid lines and dashed lines correspond to even and odd parity, respectively. In all cases $\lambda=1.0$.
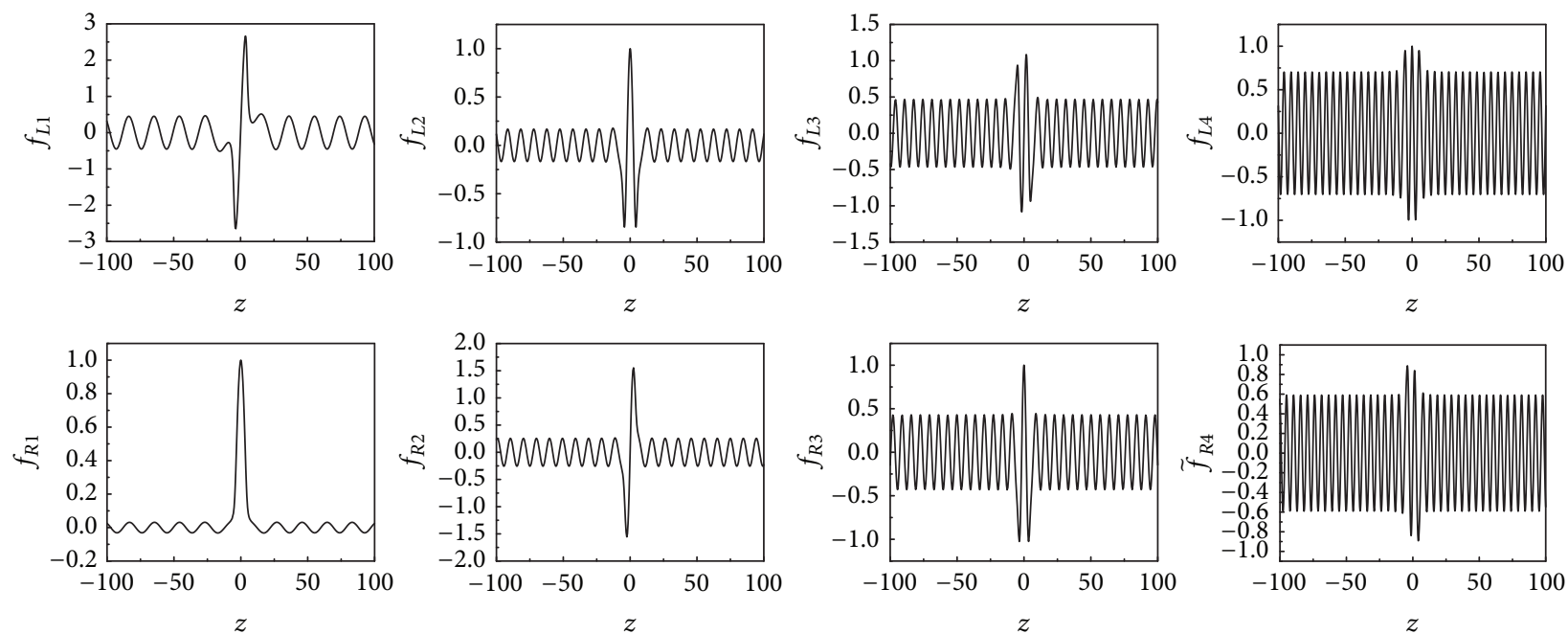

FIGURE 6: Plots of the left-handed and right-handed fermion resonances with $\eta=1, \lambda=1$, and $a=5$. 
TABLE 1: Masses of resonances for different values of $\eta$ and $a$.

(a)

\begin{tabular}{lccc}
\hline Left & $\eta=0.5$ & $\eta=1.0$ & $\eta=2.0$ \\
\hline$a=1.0$ & Absent & 1.185465 & 1.719258 \\
\hline & & & $0.689911 ;$ \\
$a=3.0$ & 0.453441 & $0.562819 ;$ & $1.232968 ;$ \\
& & 0.994057 & $1.664278 ;$ \\
& & 2.034932 \\
\hline & & & $0.377447 ;$ \\
& & $0.337121 ;$ & $0.731511 ;$ \\
& & $0.646626 ;$ & $1.058468 ;$ \\
& $0.288833 ;$ & $0.924378 ;$ & $1.359395 ;$ \\
& 0.567862 & 1.198549 & $1.831559 ;$ \\
& & & 2.146727
\end{tabular}

(b)

\begin{tabular}{llcc}
\hline Right & $\eta=0.5$ & $\eta=1.0$ & $\eta=2.0$ \\
\hline$a=1.0$ & Absent & 1.110830 & 1.711422 \\
\hline & & & $0.689911 ;$ \\
$a=3.0$ & 0.442279 & $0.562819 ;$ & $1.232968 ;$ \\
& & 0.994057 & $1.662400 ;$ \\
& & 2.027853 \\
\hline \multirow{3}{*}{0.0} & & $0.337121 ;$ & $0.731484 ;$ \\
& $0.283917 ;$ & $0.645689 ;$ & $1.058468 ;$ \\
& & $0.921857 ;$ & $1.359395 ;$ \\
& & 1.190822 & $1.631559 ;$ \\
& & & 2.139101 \\
\hline
\end{tabular}

$\tau_{4}=4.5196$ for $m_{4}=1.190822$. Therefore we can see that the lighter resonances have longer lifetimes.

\section{Conclusions}

In this paper we have studied the issue of the localization of fermion field on the double wall brane. This brane includes two scalar fields coupled minimally to brane. For observing whether the zero mode can be localized on this brane or not, we use a Yukawa coupling between fermion and background scalar fields. By investigating normalization condition, we can see that fermion couples with summation of kinks. We can see that by this coupling the zero mode of left-handed fermion can be localized on the brane under the special condition. This condition provides relation between coupling constant and $\lambda$ parameter. Also we found that the zero mode of righthanded fermion can not be localized on the brane.

The massive mode resonances were investigated numerically. From the volcano shape of effective potential for left-handed fermions, it results that the spectrum is continuous and there is no gap between zero mode and KK excitation modes. Also larger values of coupling constant and the distance of subbranes support more resonances in the spectrum. In one spectrum, heavier resonances have broader peaks rather than lighter ones. This means the lighter fermions couple stronger to the brane rather than heavier KK modes. Because of very narrow peak, we may not see light resonances. Fortunately because of supersymmetric feature of Schrödinger-like equation, we have successively even and odd parity for left or right chirality modes with the same mass in the spectrum. This helps us to search a small region when a resonance peak is not seen. Therefore numeric procedure becomes simple and fast. The lifetime of a resonance is proportional to inverse of peak width at half maximum. Hence generally, light resonances have longer lifetimes.

\section{Conflicts of Interest}

The authors declare that they have no conflicts of interest.

\section{References}

[1] L. Randall and R. Sundrum, "Large mass hierarchy from a small extra dimension," Physical Review Letters, vol. 83, no. 17, pp. 3370-3373, 1999.

[2] N. Arkani-Hamed, S. Dimopoulos, and G. Dvali, "The hierarchy problem and new dimensions at a millimeter," Physics Letters, Section B: Nuclear, Elementary Particle and High-Energy Physics, vol. 429, no. 3-4, pp. 263-272, 1998.

[3] I. Antoniadis, N. Arkani-Hamed, S. Dimopoulos, and G. R. Dvali, "New dimensions at a millimeter to a fermi and superstrings at a TeV," Physics Letters B, vol. 436, no. 3-4, pp. 257-263, 1998.

[4] V. A. Rubakov and M. E. Shaposhnikov, "Do we live inside a domain wall?" Physics Letters B, vol. 125, no. 2-3, pp. 136-138, 1983.

[5] C. Csáki, J. Erlich, C. Grojean, and T. J. Hollowood, “General properties of the self-tuning domain wall approach to the cosmological constant problem," Nuclear Physics B, vol. 584, no. 1-2, pp. 359-386, 2000.

[6] L. Randall and R. Sundrum, "An alternative to compactification," Physical Review Letters, vol. 83, no. 23, pp. 4690-4693, 1999.

[7] O. Arias, R. Cardenas, and I. Quiros, “Thick brane worlds arising from pure geometry," Nuclear Physics. B, vol. 643, no. 1-3, pp. 187-200, 2002.

[8] N. Barbosa-Cendejas and A. Herrera-Aguilar, "4D gravity localized in non Bbb $\mathbb{Z}_{2}$-symmetric thick branes," Journal of High Energy Physics, vol. 2005, no. 10, p. 101, 2005.

[9] N. Barbosa-Cendejas and A. Herrera-Aguilar, "Localization of 4D gravity on pure geometrical thick branes," Physical Review. D. Third Series, vol. 73, Article ID 084022, 2006.

[10] Y. X. Liu, K. Yang, and Y. Zhong, "de Sitter thick brane solution in Weyl geometry," Journal of High Energy Physics, vol. 2010, article 69, 2010.

[11] A. Herrera-Aguilar, D. Malagón-Morejón, and R. Rigel MoraLuna, "Localization of gravity on a de Sitter thick braneworld without scalar fields," Journal of High Energy Physics, vol. 2010, article 15, 2010.

[12] A. A. Andrianov, V. A. Andrianov, P. Giacconi, and R. Soldati, "Domain wall generation by fermion self-interaction and light particles," Journal of High Energy Physics, vol. 307, article 63, 2003.

[13] A. A. Andrianov, V. A. Andrianov, P. Giacconi, and R. Soldati, "Brane world generation by matter and gravity," Journal of High Energy Physics, vol. 7, article 3, 2005. 
[14] F. Bonjour, C. Charmousis, and R. Gregory, "Thick domain wall universes," Classical and Quantum Gravity, vol. 16, no. 7, pp. 2427-2445, 1999.

[15] W. D. Goldberger and M. B. Wise, "Modulus stabilization with bulk fields," Physical Review Letters, vol. 83, no. 24, pp. 49224925, 1999.

[16] Y. X. Liu, Y. Zhong, and K. Yang, "Scalar-kinetic branes," Europhysics Letters, vol. 90, no. 5, Article ID 51001, 2010.

[17] A. Melfo, N. Pantoja, and A. Skirzewski, "Thick domain wall spacetimes with and without reflection symmetry," Physical Review D, vol. 67, no. 10, Article ID 105003, 2003.

[18] A. E. Chumbes and M. B. Hott, "Nonpolynomial potentials with deformable topological structures," Physical Review D, vol. 81, no. 4, Article ID 045008, 2010.

[19] R. Gregory and A. Padilla, "Braneworld instantons," Classical and Quantum Gravity, vol. 19, no. 2, pp. 279-302, 2002.

[20] M. Gremm, "Four-dimensional gravity on a thick domain wall," Physics Letters B, vol. 478, no. 4, pp. 434-438, 2000.

[21] S. Kobayashi, K. Koyama, and J. Soda, "Thick brane worlds and their stability," Physical Review D, vol. 65, no. 6, Article ID 064014, 2002.

[22] M. Peyrard and M. D. Kruskal, "Kink dynamics in the highly discrete sine-Gordon system," Physica D: Nonlinear Phenomena, vol. 14, no. 1, pp. 88-102, 1984.

[23] A. Ahmed and B. Grzadkowski, "Brane modeling in warped extra-dimension," Journal of High Energy Physics, vol. 2013, article 177, 2013.

[24] A. de Souza Dutra, G. P. de Brito, and J. M. Hoff da Silva, "Asymmetrical bloch branes and the hierarchy problem," epl-A Letters Journals Exploring The Frontiers of Physics, vol. 108, no. 1, p. 11001, 2014.

[25] J. Khoury, B. A. Ovrut, P. J. Steinhardt, and N. Turok, "Ekpyrotic universe: colliding branes and the origin of the hot big bang," Physical Review D, vol. 64, no. 12, Article ID 123522, 2001.

[26] R. Kallosh, L. Kofman, and A. Linde, "Pyrotechnic universe," Physical Review D, vol. 64, no. 12, Article ID 123523, 2001.

[27] M. Bucher, "A braneworld universe from colliding bubbles," Physics Letters B, vol. 530, no. 1-4, pp. 1-9, 2002.

[28] U. Gen, A. Ishibashi, and T. Tanaka, "Brane big bang brought on by a bulk bubble," Physical Review D, vol. 66, no. 2, Article ID 023519, 2002.

[29] A. Campos, "Critical phenomena of thick branes in warped spacetimes," Physical Review Letters, vol. 88, no. 14, Article ID 141602, 2002.

[30] J. Wang, J. Wang, W. Li, and G. Liu, "Confinement induced by fermion damping in three-dimensional QED," Physical Review D, vol. 82, no. 6, Article ID 067701, 2010.

[31] D. Bazeia, J. Menezes, and R. Menezes, "New global defect structures," Physical Review Letters, vol. 91, no. 24, Article ID 241601, 2003.

[32] D. Bazeia and L. Losano, "Deformed defects with applications to braneworlds," Physical Review D, vol. 73, no. 2, Article ID 025016, 2006.

[33] D. Bazeia, H. Boschi-Filho, and F. A. Brito, "Domain defects in systems of two real scalar fields," Journal of High Energy Physics, vol. 1999, no. 4, article 28, 1999.

[34] A. d. Dutra, G. P. de Brito, and J. M. da Silva, "Method for obtaining thick brane models," Physical Review D. Particles, Fields, Gravitation, and Cosmology, vol. 91, no. 8, Article ID 086016, 2015.
[35] B. Bajc and G. Gabadadze, "Localization of matter and cosmological constant on a brane in anti de Sitter space," Physics Letters. B, vol. 474, no. 3-4, pp. 282-291, 2000.

[36] I. Oda, "Localization of matters on a string-like defect," Physics Letters B, vol. 496, no. 1-2, pp. 113-121, 2000.

[37] S. Randjbar-Daemi and M. E. Shaposhnikov, "Fermion zeromodes on brane-worlds," Physics Letters B, vol. 492, no. 3-4, pp. 361-364, 2000.

[38] Y. Grossman and M. Neubert, "Neutrino masses and mixings in non-factorizable geometry," Physics Letters. B, vol. 474, no. 3-4, pp. 361-371, 2000.

[39] Y. X. Liu, X. H. Zhang, L. D. Zhang, and Y. S. Duan, "Localization of matters on pure geometrical thick branes," Journal of High Energy Physics, vol. 2008, no. 2, p. 67, 2008.

[40] R. Koley and S. Kar, "A novel braneworld model with a bulk scalar field," Physics Letters B, vol. 623, no. 3-4, pp. 244-250, 2005, Erratum: [Physics Letters B, vol. 631, pp. 199, 2005].

[41] A. Melfo, N. Pantoja, and J. D. Tempo, "Fermion localization on thick branes," Physical Review. D. Third Series, vol. 73, Article ID 044033, 2006.

[42] S. Ichinose, "Fermions in kaluza-klein and randall-sundrum theories," Physical Review. D. Third Series, vol. 66, no. 10, Article ID 104015, 2002.

[43] R. Koley, J. Mitra, and S. SenGupta, "Chiral fermions in a spacetime with multiple warping," Physical Review D. Particles, Fields, Gravitation, and Cosmology, vol. 78, no. 4, 045005, 6 pages, 2008.

[44] Y. Liu, L. Zhang, S. Wei, and Y. Duan, "Localization and mass spectrum of matters on Weyl thick branes," Journal of High Energy Physics, vol. 2008, 2008.

[45] M. Gogberashvili, A. Herrera-Aguilar, D. Malagón-Morejón, R. R. Mora-Luna, and U. Nucamendi, "Thick brane isotropization in a generalized 5D anisotropic standing wave braneworld model," Physical Review D, vol. 87, no. 8, Article ID 084059, 2013.

[46] Z.-H. Zhao, Y.-X. Liu, and H.-T. Li, "Fermion localization on asymmetric two-field thick branes," Classical and Quantum Gravity, vol. 27, no. 18, 2010.

[47] L. B. Castro, "Fermion localization on two-field thick branes," Physical Review D, vol. 83, no. 4, Article ID 045002, 2011.

[48] C. A. Almeida, R. Casana, J. Ferreira, and A. R. Gomes, "Fermion localization and resonances on two-field thick branes," Physical Review D. Particles, Fields, Gravitation, and Cosmology, vol. 79, no. 12, Article ID 125022, 2009.

[49] Y. X. Liu, C. E. Fu, L. Zhao, and Y. S. Duan, "Localization and mass spectra of fermions on symmetric and asymmetric thick branes," Physical Review D, vol. 80, no. 6, Article ID 065020, 2009.

[50] Y. X. Liu, J. Yang, Z. H. Zhao, C. E. Fu, and Y. S. Duan, "Fermion localization and resonances on a de Sitter thick brane," Physical Review D, vol. 80, Article ID 065019, 2009. 

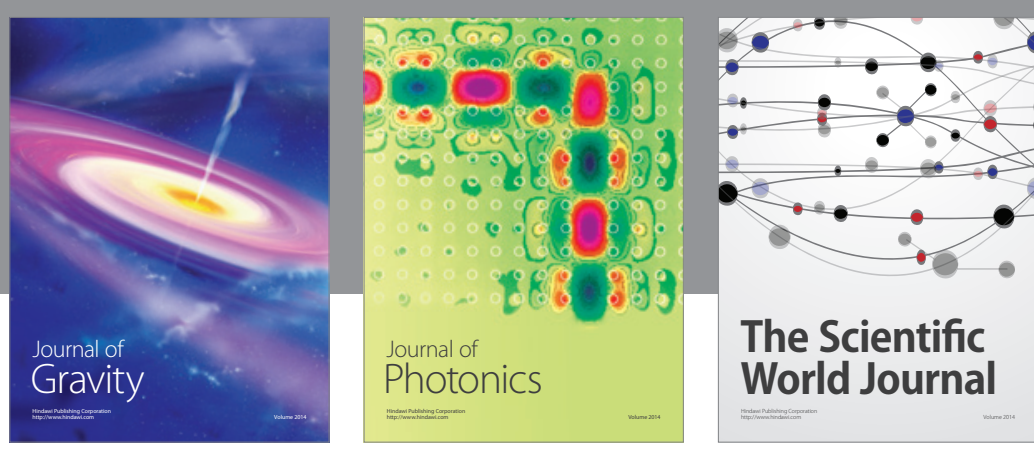

The Scientific World Journal
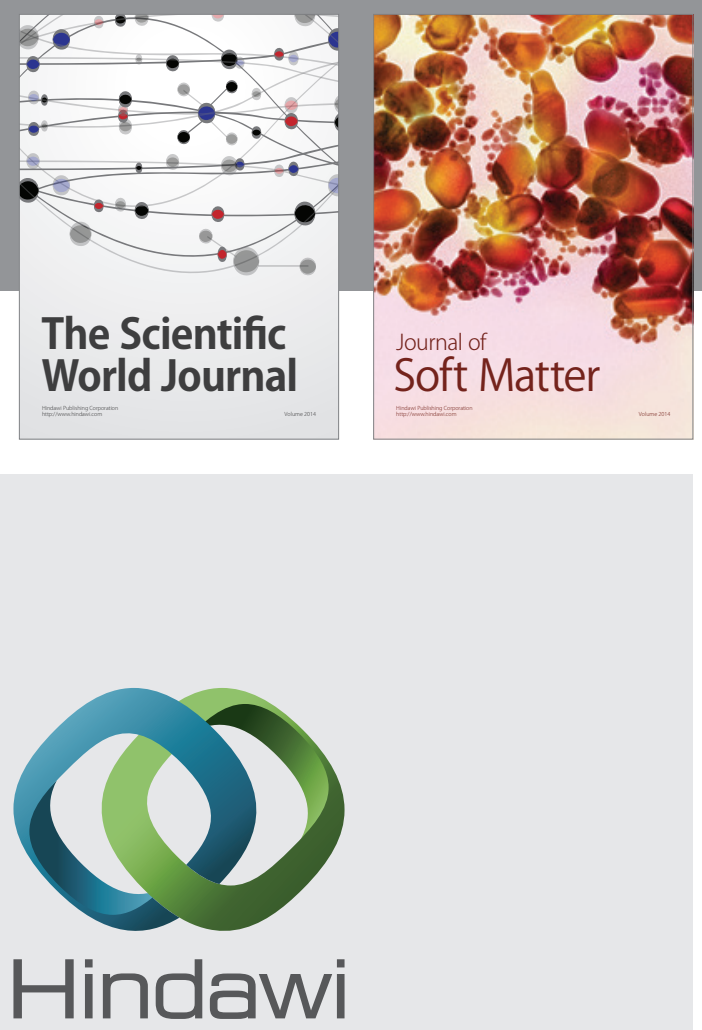

Submit your manuscripts at

https://www.hindawi.com
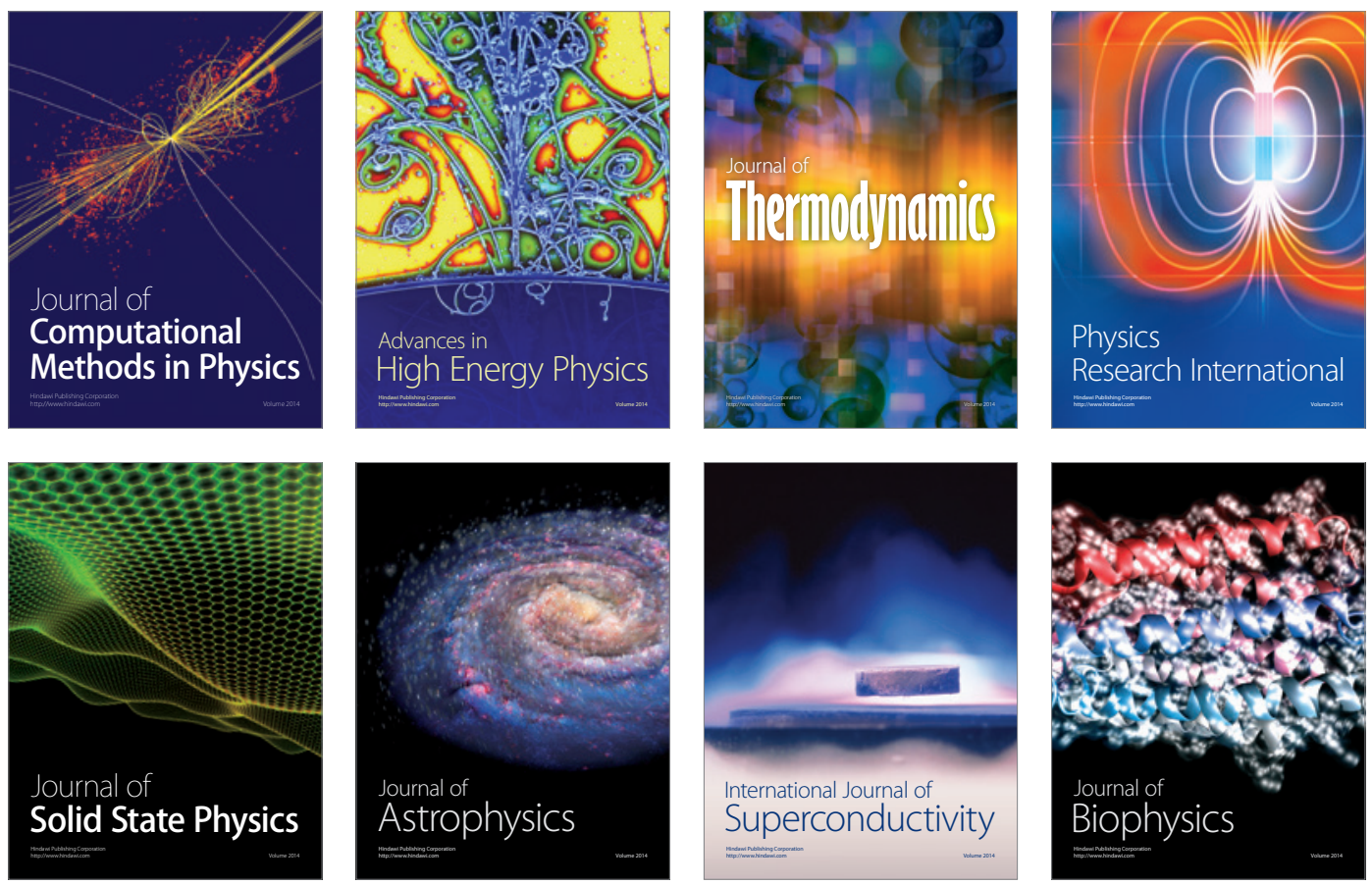
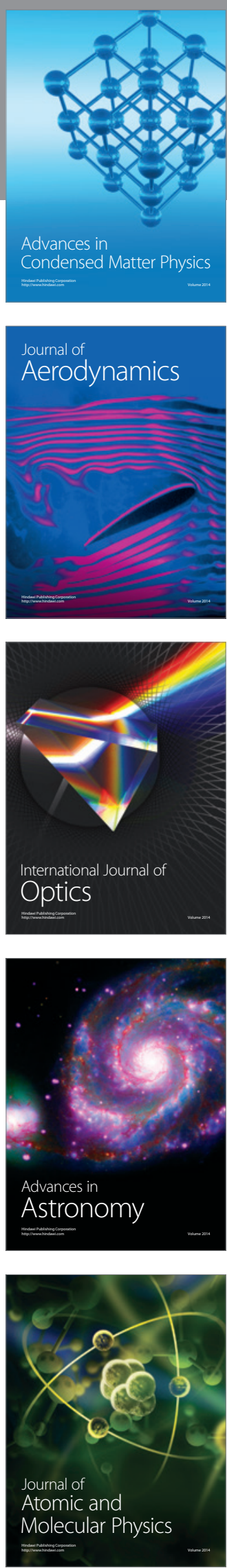